\title{
Quark orbital angular momentum in the proton evaluated using a direct derivative method
}

M. Engelhardt ${ }^{* a \dagger}$, J. Green ${ }^{b}$, N. Hasan ${ }^{c, d}$, S. Krieg ${ }^{c, d}$, S. Meinel ${ }^{e, f}$, J. Negele ${ }^{g}$, A. Pochinsky ${ }^{g}$ and S. Syritsyn ${ }^{f, h}$

${ }^{a}$ Department of Physics, New Mexico State University, Las Cruces, NM 88003, USA

${ }^{b}$ NIC, Deutsches Elektronen-Synchroton, 15738 Zeuthen, Germany

${ }^{c}$ Bergische Universität Wuppertal, 42119 Wuppertal, Germany

${ }^{d}$ IAS, Jülich Supercomputing Centre, Forschungszentrum Jülich, 52425 Jülich, Germany

${ }^{e}$ Department of Physics, University of Arizona, Tucson, AZ 85721, USA

${ }^{f}$ RIKEN BNL Research Center, Brookhaven National Laboratory, Upton, NY 11973, USA

${ }^{g}$ Center for Theoretical Physics, Massachusetts Institute of Technology, Cambridge, MA 02139, USA

${ }^{h}$ Department of Physics and Astronomy, Stony Brook University, Stony Brook, NY 11794, USA

${ }^{\dagger} E$-mail: engel@nmsu.edu

Quark orbital angular momentum (OAM) in the proton can be calculated directly given a Wigner function encoding the simultaneous distribution of quark transverse positions and momenta. This distribution can be accessed via proton matrix elements of a quark bilocal operator (the separation in which is Fourier conjugate to the quark momentum) featuring a momentum transfer (which is Fourier conjugate to the quark position). To generate the weighting by quark transverse position needed to calculate OAM, a derivative with respect to momentum transfer is consequently required. This derivative is evaluated using a direct derivative method, i.e., a method in which the momentum derivative of a correlator is directly sampled in the lattice calculation, as opposed to extracting it a posteriori from the numerical correlator data. The method removes the bias stemming from estimating the derivative a posteriori that was seen to afflict a previous exploratory calculation. Data for Ji OAM generated on a clover ensemble at pion mass $m_{\pi}=317 \mathrm{MeV}$ are seen to agree with the result obtained via the traditional Ji sum rule method. By varying the gauge connection in the quark bilocal operator, also Jaffe-Manohar OAM is extracted, and seen to be enhanced significantly compared to Ji OAM.

23rd International Spin Physics Symposium - SPIN2018 -

10-14 September, 2018

Ferrara, Italy

* Speaker. 


\section{Introduction}

A prominent endeavor in the study of hadron structure is understanding the decomposition of the spin of the proton into the contributions from the spins and orbital angular momenta (OAM) of its quark and gluon constituents. Since gauge invariance prevents a consideration of quark and gluon degrees of freedom in isolation, there is no unique definition of quark OAM; any such definition will contain gluonic effects to a varying degree. Two widely studied decomposition schemes are the ones due to Ji [1] and to Jaffe and Manohar [2].

Recently, a method to evaluate quark OAM in Lattice QCD directly from the simultaneous distribution of quark transverse positions and momenta in a rapidly propagating proton was explored in [3]. This distribution is encoded in generalized transverse momentum-dependent parton distributions (GTMDs) [4-7]; in comparison to standard TMDs, which parametrize forward matrix elements of an appropriate bilocal quark operator, GTMDs additionally include a momentum transfer. The momentum transfer is Fourier conjugate to the quark impact parameter and thus supplements the transverse momentum information with transverse position information, generating, in effect, a Wigner function. The freedom in choosing the gauge connection in the bilocal quark operator allows one to access a continuum of quark OAM definitions, including the ones of Ji and Jaffe-Manohar. In this respect, the GTMD approach extends beyond the standard quark OAM calculation via Ji's sum rule, which yields specifically Ji OAM.

The present work constitutes a further methodological development of the approach introduced in [3]. The result for Ji OAM obtained using the concrete implementation in [3] deviated significantly from the standard Ji sum rule result, due to a systematic bias inherent in the numerical method. This discrepancy is resolved in the present work, validating the GTMD approach. Furthermore, the present investigation is carried out at a significantly lower pion mass, $m_{\pi}=317 \mathrm{MeV}$.

\section{Quark orbital angular momentum}

The quark OAM component $L_{3}^{U}$ in a longitudinally polarized proton propagating in the 3direction can be accessed via a GTMD matrix element [5],

$$
L_{3}^{U}=\left.\frac{1}{2 P^{+}} \varepsilon_{i j} \frac{\partial}{\partial z_{T, i}} \frac{\partial}{\partial \Delta_{T, j}} \frac{\left\langle p^{\prime}, S^{\prime}=\vec{e}_{3}\left|\bar{\psi}(-z / 2) \gamma^{+} U \psi(z / 2)\right| p, S=\vec{e}_{3}\right\rangle}{\mathscr{S}[U]}\right|_{z^{+}=z^{-}=0, \Delta_{T}=0, z T \rightarrow 0}
$$

A number of remarks are in order concerning this expression. The initial and final proton momenta are treated symmetrically, $p=P-\Delta_{T} / 2, p^{\prime}=P+\Delta_{T} / 2$, where the spatial component of $P$ is in 3-direction and the momentum transfer $\Delta_{T}$ is transverse. Since $\Delta_{T}$ is Fourier conjugate to the quark impact parameter $b_{T}$, evaluating the $\Delta_{T}$-derivative at $\Delta_{T}=0$ amounts to averaging $b_{T}$. On the other hand, the transverse quark operator separation $z_{T}$ is Fourier conjugate to the transverse quark momentum $k_{T}$; therefore, evaluating the $z_{T}$-derivative at $z_{T}=0$ amounts to averaging $k_{T}$. Here, the limit $z_{T} \rightarrow 0$ must be taken carefully, since it is associated with ultraviolet divergences. In aggregate, thus, (2.1) yields the average $b_{T} \times k_{T}$, i.e., OAM in the 3-direction. Also the longitudinal quark momentum components are integrated over in view of the specification $z^{+}=z^{-}=0$. In the thus constructed average, quark spin direction is immaterial owing to the use of the Dirac structure $\gamma^{+}$. Finally, (2.1) depends on the gauge link $U$ connecting the quark operators, along with a soft 
factor $\mathscr{S}[U]$ which absorbs divergences associated with the quantum fluctuations of $U$; for present purposes, one may consider $\mathscr{S}[U]$ to include also renormalization factors associated with the quark field operators. This soft factor is the same as for the standard TMD matrix element [8], since (2.1) only differs from the latter in the external state, not the operator. The multiplicative factor $\mathscr{S}[U]$ will be canceled by forming an appropriate ratio below and thus does not need to be specified in more detail. It is in the path of $U$ that different definitions of quark OAM are encoded; (2.1) is a functional of $U$. In the present work, staple-shaped $U \equiv U[-z / 2, \eta v-z / 2, \eta v+z / 2, z / 2]$ are considered, where the arguments of $U$ are positions joined by straight Wilson lines. Thus, the vector $v$ gives the direction of the staple, and the length of the staple is scaled by the parameter $\eta$. For $\eta=0$, one has a straight Wilson line directly connecting the quark operators.

The $\eta=0$ straight gauge link limit corresponds to Ji OAM [9], whereas the $\eta \rightarrow \pm \infty$ limit of a staple extending to infinity yields Jaffe-Manohar OAM [10]. Such a staple link incorporates final state interactions, e.g., in semi-inclusive deep inelastic scattering (SIDIS) processes, with the staple legs corresponding to the direction of propagation of the struck quark. Thus, Jaffe-Manohar quark OAM differs from Ji quark OAM in that it includes the integrated torque accumulated by the struck quark as it leaves the proton [11]. In a Lattice QCD calculation, $\eta$ can be varied quasi-continuously, with the Jaffe-Manohar limit achieved by extrapolation. This yields a gauge-invariant interpolation between the Ji and Jaffe-Manohar cases.

In addition, the direction $v$ of the staple needs to be specified. The most straightforward choice for the direction of propagation of the struck quark in a hard scattering process would initially appear to be a lightlike vector. However, such a choice leads to severe rapidity divergences, which are regulated in the scheme advanced in $[12,13]$ by taking $v$ off the light cone into the spacelike region. The matrix element (2.1) determining quark OAM therefore depends on the additional Collins-Soper type parameter $\hat{\zeta}=v \cdot P /\left(\sqrt{\left|v^{2}\right|} \sqrt{P^{2}}\right)$. The light-cone limit corresponds to $\hat{\zeta} \rightarrow \infty$.

As in lattice TMD studies [14-16], an appropriate ratio of quantities can be employed to cancel the soft factor $\mathscr{S}[U]$. A suitable quantity for this purpose is the number of valence quarks

$$
n=\left.\frac{1}{2 P^{+}} \frac{\left\langle p^{\prime}, S^{\prime}=\vec{e}_{3}\left|\bar{\psi}(-z / 2) \gamma^{+} U \psi(z / 2)\right| p, S=\vec{e}_{3}\right\rangle}{\mathscr{S}[U]}\right|_{z^{+}=z^{-}=0, \Delta_{T}=0, z_{T} \rightarrow 0}
$$

which only differs from (2.1) by omitting the weighting with $b_{T} \times k_{T}$ (in terms of the Fourier conjugate variables), and thus counts quarks. The soft factor $\mathscr{S}[U]$ is even in $z_{T}$, and thus cancels when forming the ratio $L_{3}^{U} / n$. Furthermore, at finite lattice spacing $a$, the derivative with respect to $z_{T}$ in (2.1) is realized as a finite difference, leading to the renormalized quantity evaluated in practice,

$$
\frac{L_{3}^{U}}{n}=\left.\frac{1}{a} \varepsilon_{i j} \frac{\frac{\partial}{\partial \Delta_{T, j}}\left(\Phi\left(a \vec{e}_{i}\right)-\Phi\left(-a \vec{e}_{i}\right)\right)}{\Phi\left(a \vec{e}_{i}\right)+\Phi\left(-a \vec{e}_{i}\right)}\right|_{z^{+}=z^{-}=0, \Delta_{T}=0}
$$

where summation over the transverse indices $i$ and $j$ is implied, and the abbreviation $\Phi\left(z_{T}\right)=$ $\left\langle p^{\prime}, S^{\prime}=\vec{e}_{3}\left|\bar{\psi}(-z / 2) \gamma^{+} U \psi(z / 2)\right| p, S=\vec{e}_{3}\right\rangle$ has been introduced. On the other hand, (2.3) also calls for a derivative with respect to $\Delta_{T}$. In the initial exploration [3], this derivative was likewise realized as finite difference. This led to a significant systematic bias in the numerical results because of the substantial increment in $\Delta_{T}$ employed. The chief advance of the present study is to evaluate this derivative using a direct derivative method, as described below. The present study furthermore is carried out at a lower pion mass, $m_{\pi}=317 \mathrm{MeV}$, than used in [3]. 


\section{Direct derivative method}

The principal ingredient needed to extract the matrix element $\Phi\left(z_{T}\right)$ is the following threepoint correlator, constructed using proton sources and $\operatorname{sinks} \bar{N}, N$, projected onto proton momentum $P+\Delta_{T} / 2$ at the sink and onto momentum transfer $\Delta_{T}$ at the operator insertion, as well as onto longitudinal polarization, encoded in $\Gamma_{\text {pol }}$,

$$
\begin{aligned}
C_{3} & =\operatorname{Tr}\left[\sum_{x, y} e^{-i\left(P+\Delta_{T} / 2\right) \cdot(x-y)} e^{-i\left(P-\Delta_{T} / 2\right) \cdot y}\left\langle N(x) \bar{\psi}(y-z / 2) \gamma^{+} U \psi(y+z / 2) \bar{N}(0)\right\rangle \Gamma_{\mathrm{pol}}\right] \\
& =\sum_{x, y} e^{-i P \cdot x}\left\langle\operatorname{Tr}\left[\left(\gamma_{5} G_{\mathrm{pt}-\mathrm{sm}}\left(y-z / 2, x, \Delta_{T} / 2\right) \gamma_{5} S_{\Gamma_{\mathrm{pol}}}^{N \bar{N}^{\dagger}}(0 ; x)\right)^{\dagger} \gamma^{+} U G_{\mathrm{pt}-\mathrm{sm}}\left(y+z / 2,0,-\Delta_{T} / 2\right)\right]\right\rangle
\end{aligned}
$$

Here, the second line corresponds to the standard evaluation of the correlator through a sequential source, $S_{\Gamma_{\mathrm{pol}}}^{N \bar{N}}(0 ; x)$, in which, however, the phases associated with the projection onto momentum transfer $\Delta_{T}$ have been absorbed into the propagators, $G_{\mathrm{pt}-\mathrm{sm}}(s, t, q)=e^{-i q \cdot(s-t)} G_{\mathrm{pt}-\mathrm{sm}}(s, t)$, where $G_{\mathrm{pt-sm}}(s, t)$ denotes the standard smeared-to-point propagator. In this form, the dependence on $\Delta_{T}$ resides purely in the (modified) propagators, and the derivative of the correlator with respect to $\Delta_{T}$ can be assembled once one has constructed the derivatives of the propagators. The derivative of a modified point-to-point propagator $G$ is discussed in detail in [17]; essentially, the derivative, evaluated at $\Delta_{T}=0$, generates a vector current insertion into the propagator. Generalized to smeared-to-point propagators, which contain an additional convolution with a smearing kernel $K$, one obtains a further term from the derivative of $K$ [18],

$$
\left.\frac{\partial}{\partial q_{j}} G_{\mathrm{pt}-\mathrm{sm}}(s, t, q)\right|_{q=0}=\sum_{x} G(s, x)\left[-i \sum_{y} V_{j} G(x, y) K(y, t)+\left.\frac{\partial}{\partial q_{j}} e^{-i q \cdot(x-t)} K(x, t)\right|_{q=0}\right]
$$

where $V_{j}$ is the conserved vector current insertion operator. The computation of the derivative of the smearing kernel is discussed in detail in [18]. By constructing and evaluating correlators corresponding directly to derivatives of $C_{3}$, cf. (3.1), in this fashion, any systematic bias in carrying out the derivative with respect to $\Delta_{T}$ in (2.3) is avoided.
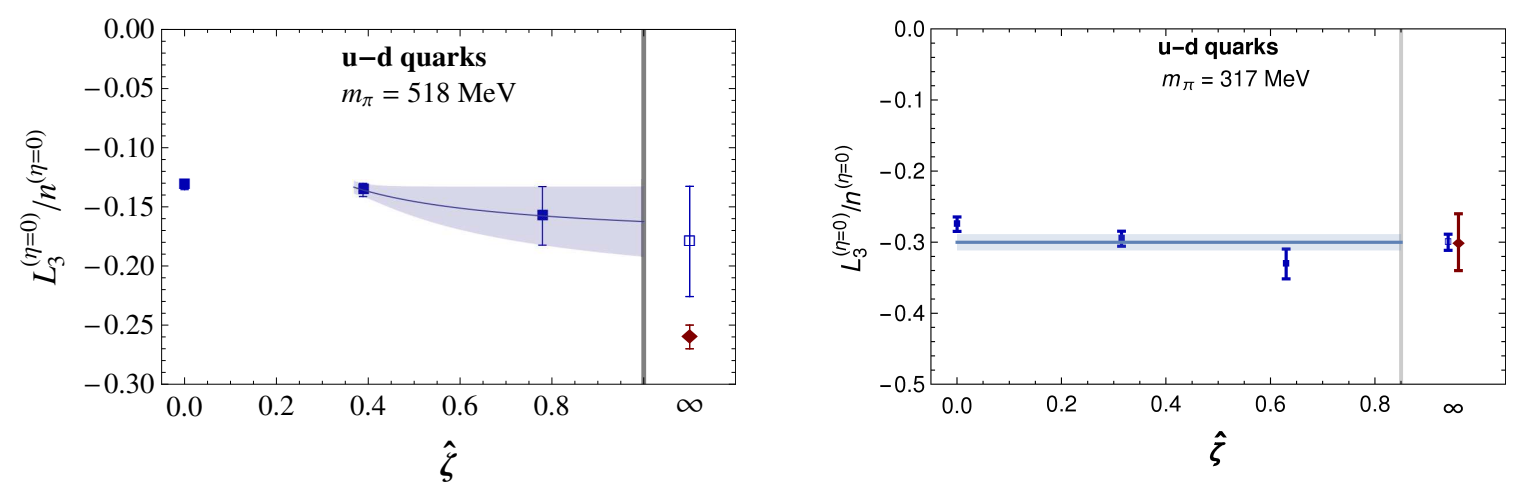

Figure 1: Ji quark OAM obtained for different $\hat{\zeta}$, with extrapolations to large $\hat{\zeta}$ (blue squares), compared to the Ji sum rule result (red diamonds). The left panel is taken from [3], the right panel displays the results of the present work. 


\section{Lattice calculation and results}

To perform a lattice calculation of the ratio (2.3), the problem must be boosted into a Lorentz frame in which the TMD operator entering $\Phi\left(z_{T}\right)$ exists at a single time. There is no obstacle to this, given that the directions of $z$ and $v$ are both spacelike, cf. the discussion above preceding eq. (2.2). In the frame preferred for the lattice calculation, $v$ points in the longitudinal 3-direction, whereas $z_{T}$ is transverse, in the direction orthogonal to the momentum transfer $\Delta_{T}$. In this frame, $\Phi\left(z_{T}\right)$ can be evaluated using standard Lattice QCD methods. Numerical data for the ratio (2.3) were obtained on a clover fermion ensemble constituted of $32^{3} \times 96$ lattices with spacing $a=0.114 \mathrm{fm}$ and pion mass $m_{\pi}=317 \mathrm{MeV}$. The source-sink separation employed was $10 a=1.14 \mathrm{fm}$. The longitudinal
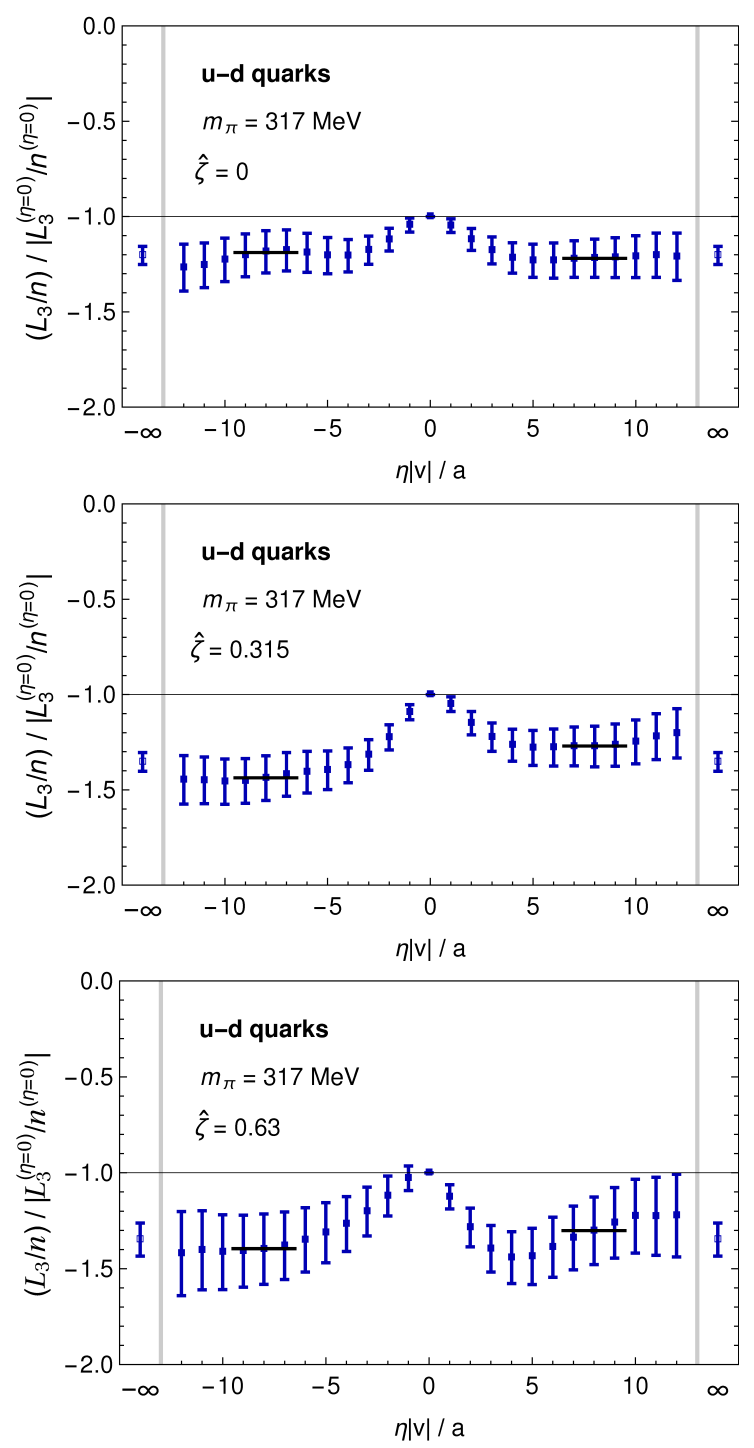

Figure 2: Quark OAM as a function of staple length $\eta$, normalized to the modulus of the $\eta=0 \mathrm{Ji}$ OAM value. Asymptotic values were extracted by averaging over data at $\eta|v| / a= \pm 7, \pm 8, \pm 9$. proton momentum components $P_{3}=0$, $2 \pi /(a L), 4 \pi /(a L)$ were included in the calculation, where $L=32$ denotes the spatial lattice extent. This corresponds to CollinsSoper parameters $\hat{\zeta}=0,0.315,0.63$.

Examining first the $\eta=0$ limit, corresponding to Ji OAM, Fig. 1 compares the results obtained in the present calculation (right panel) with results taken from the initial exploration [3] (left panel). Data for three values of $\hat{\zeta}$ are displayed, together with an extrapolation to large $\hat{\zeta}$, compared to Ji OAM as obtained via Ji's sum rule at the same pion mass [19]. It should be noted that, by maintaining the definition of $v$ as pointing in the longitudinal 3-direction, $\hat{\zeta}$ can still formally be defined in the $\eta=0$ case, and it characterizes the momentum of the proton; however, Ji OAM ultimately cannot depend on this parameter, since $v$ does not enter the definition of the straight gauge link. I.e., Ji OAM is boost-invariant.

This constraint was not taken into account in the extrapolation of the data in the left panel [3], which themselves are compatible with constant behavior. Instead, an ad hoc fit allowing for an approach to large $\hat{\zeta}$ proportional to $1 / \hat{\zeta}$ was performed. As a result, the central value and uncertainty of the extrapolation extend to larger magnitudes than they would by fitting a constant; with the latter fit, the discrepancy with the $\mathrm{Ji}$ sum rule value would be exhibited more 


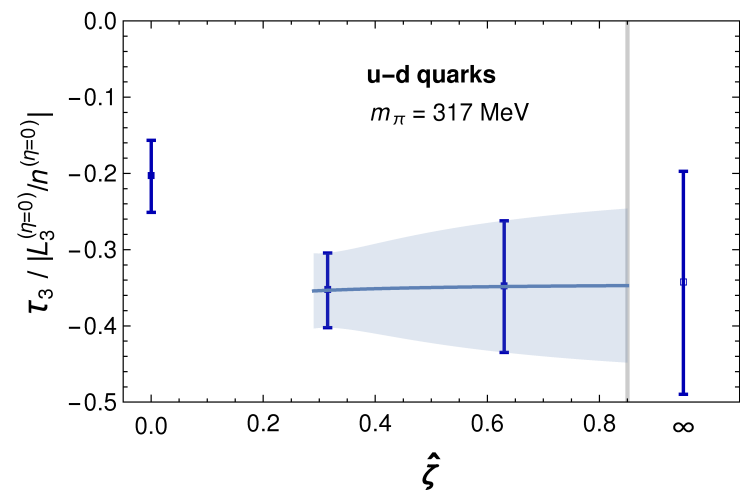

Figure 3: Torque accumulated by the struck quark, cf. main text, normalized to the modulus of the $\eta=0 \mathrm{Ji}$ OAM value. starkly. As already noted above, this discrepancy is owed to the biased estimate of the $\Delta_{T}$-derivative in (2.3) via a finite difference employed in [3].

By contrast, the present calculation, cf. the right panel in Fig. 1, treats the $\Delta_{T}$ derivative in (2.3) in an unbiased manner, and is seen to agree well with the Ji sum rule result for all three proton momenta, as well as when extrapolated to large $\hat{\zeta}$ using a constant fit. This verifies that the discrepancy observed in the initial study [3] indeed was due to the biased estimate of the $\Delta_{T}-$ derivative. The unbiased treatment via the

direct derivative method removes the discrepancy. The evaluation of Ji OAM via the nonlocal GTMD matrix element (2.1) coincides with the evaluation via the local matrix elements encoding the GPD moments entering Ji's sum rule, validating the GTMD method.

Turning to the transition from Ji OAM to Jaffe-Manohar OAM as a function of the staple length $\eta$, Fig. 2 displays data at the three different available $\hat{\zeta}$. The data are normalized to the magnitude of the $\eta=0 \mathrm{Ji}$ value. Starting with Ji quark OAM at $\eta=0$, the struck quark in a deep inelastic scattering process accumulates torque as it is leaving the proton, to finally end up with Jaffe-Manohar OAM at large $\eta$. The effect is substantial, can be clearly resolved in the data, and is directed such as to enhance the magnitude of OAM compared to the $\eta=0$ value. It increases as one departs from the $\hat{\zeta}=0$ limit towards finite proton momenta; no significant difference between the results at the two nonvanishing $\hat{\zeta}$ is seen. The effect is thus likely to survive the extrapolation to large $\hat{\zeta}$. Fig. 3 displays such an extrapolation for the integrated torque $\tau_{3}=$ $L_{3}^{(\eta=\infty)} / n^{(\eta=\infty)}-L_{3}^{(\eta=0)} / n^{(\eta=0)}$ alone, using the fit ansatz $A+B / \hat{\zeta}$. The extrapolated integrated torque is roughly one third of the originally present Ji quark OAM in the ensemble used here.

\section{Conclusion}

The main thrust of the work presented here was the further methodological development of the GTMD approach to evaluating quark OAM in the proton in Lattice QCD. Specifically, eq. (2.3) calls for a derivative with respect to momentum transfer $\Delta_{T}$. Employing a direct derivative method to evaluate (2.3) free of systematic bias, the result for Ji quark OAM was seen to agree with the result obtained using the standard Ji sum rule method. This stands in contrast to the initial exploration [3], in which a biased evaluation of the $\Delta_{T}$-derivative led to a significant discrepancy in the Ji quark OAM results. The agreement achieved using the improved methodology validates the GTMD approach. Furthermore, by varying the gauge connection in the quark bilocal operator under consideration, also Jaffe-Manohar OAM was extracted, and seen to be enhanced significantly compared to Ji OAM, by about one third, at the pion mass $m_{\pi}=317 \mathrm{MeV}$ employed in this study. Going forward, the exploration of quark OAM evolution through calculations at varying lattice spacings is of interest, and investigations at lower pion masses must be pursued. 


\section{Acknowledgments}

This work benefited from fruitful discussions with M. Burkardt, S. Liuti and B. Musch. Computations were performed using resources provided by the U.S. DOE Office of Science through the National Energy Research Scientific Computing Center (NERSC), a DOE Office of Science User Facility, under Contract No. DE-AC02-05CH11231, as well as through facilities of the USQCD Collaboration at Fermilab, employing the Chroma [20] and Qlua software suites. R. Edwards, B. Joó and K. Orginos are acknowledged for providing the clover ensemble analyzed in this work, which was generated using resources provided by XSEDE (supported by National Science Foundation Grant No. ACI-1053575). S.M. is supported by the U.S. DOE, Office of Science, Office of High Energy Physics under Award Number DE-SC0009913. S.S. and S.M. also acknowledge support by the RHIC Physics Fellow Program of the RIKEN BNL Research Center. M.E., J.N., and A.P. are supported by the U.S. DOE, Office of Science, Office of Nuclear Physics through grants numbered DE-FG02-96ER40965, DE-SC-0011090 and DE-FC02-06ER41444 respectively. This work was furthermore supported by the U.S. DOE through the TMD Topical Collaboration.

\section{References}

[1] X. Ji, Phys. Rev. Lett. 78, 610 (1997).

[2] R. Jaffe and A. Manohar, Nucl. Phys. B337, 509 (1990).

[3] M. Engelhardt, Phys. Rev. D 95, 094505 (2017).

[4] S. Meißner, A. Metz and M. Schlegel, JHEP 0908, 056 (2009).

[5] C. Lorcé and B. Pasquini, Phys. Rev. D 84, 014015 (2011).

[6] A. Rajan, A. Courtoy, M. Engelhardt and S. Liuti, Phys. Rev. D 94, 034041 (2016).

[7] A. Rajan, M. Engelhardt and S. Liuti, Phys. Rev. D 98, 074022 (2018).

[8] M. Echevarria, A. Idilbi, K. Kanazawa, C. Lorcé, A. Metz, B. Pasquini and M. Schlegel, Phys. Lett. B759, 336 (2016).

[9] X. Ji, X. Xiong and F. Yuan, Phys. Rev. Lett. 109, 152005 (2012).

[10] Y. Hatta, Phys. Lett. B708, 186 (2012).

[11] M. Burkardt, Phys. Rev. D 88, 014014 (2013).

[12] S. M. Aybat and T. Rogers, Phys. Rev. D 83, 114042 (2011).

[13] J. C. Collins, Foundations of Perturbative QCD (Cambridge University Press, 2011).

[14] B. Musch, P. Hägler, M. Engelhardt, J. Negele and A. Schäfer, Phys. Rev. D 85, 094510 (2012).

[15] M. Engelhardt, P. Hägler, B. Musch, J. Negele and A. Schäfer, Phys. Rev. D 93, 054501 (2016).

[16] B. Yoon, M. Engelhardt, R. Gupta, T. Bhattacharya, J. Green, B. Musch, J. Negele, A. Pochinsky, A. Schäfer, and S. Syritsyn, Phys. Rev. D 96, 094508 (2017).

[17] G. M. de Divitiis, R. Petronzio and N. Tantalo, Phys. Lett. B718, 589 (2012).

[18] N. Hasan, J. Green, S. Meinel, M. Engelhardt, S. Krieg, J. Negele, A. Pochinsky and S. Syritsyn, Phys. Rev. D 97, 034504 (2018).

[19] J. D. Bratt et al. [LHP Collaboration], Phys. Rev. D 82, 094502 (2010).

[20] R. Edwards and B. Joó [SciDAC Collaboration], Nucl. Phys. Proc. Suppl. 140, 832 (2005). 